\title{
The High-Resolution Coronal Imager, Flight 2.1
}

Laurel A. Rachmeler $^{1}$ - Amy R. Winebarger ${ }^{1}$ - Sabrina L. Savage ${ }^{1}$. Leon Golub $^{2}$ - Ken Kobayashi ${ }^{1}$. Genevieve D. Vigil ${ }^{3}$ - David H. Brooks ${ }^{4}$ Jonathan W. Cirtain ${ }^{5}$ - Bart De Pontieu ${ }^{6,7,8}$ - David E. McKenzie ${ }^{1}$. Richard J. Morton' ${ }^{\text {D }} \cdot$ Hardi Peter $^{10}$ - Paola Testa ${ }^{2}$ (D) Sanjiv K. Tiwari ${ }^{6,11}$ (D) Robert W. Walsh ${ }^{12}$ - Harry P. Warren ${ }^{13}$ (D) Caroline Alexander ${ }^{3}$. Darren Ansell ${ }^{12}$. Brent L. Beabout ${ }^{1}$ - Dyana L. Beabout ${ }^{1}$. Christian W. Bethge ${ }^{3}$. Patrick R. Champey ${ }^{1}$. Peter N. Cheimets ${ }^{2} \cdot$ Mark A. Cooper ${ }^{1}$ - Helen K. Creel ${ }^{1} \cdot$ Richard Gates $^{2}$. Carlos Gomez $^{1}$ - Anthony Guillory ${ }^{1}$ - Harlan Haight ${ }^{1}$ - William D. Hogue ${ }^{1}$. Todd Holloway $^{1}$ - David W. Hyde ${ }^{1}$ - Richard Kenyon ${ }^{12}$ - Joseph N. Marshall ${ }^{1}$. Jeff E. McCracken ${ }^{1} \cdot$ Kenneth McCracken $^{2}$ - Karen O. Mitchell ${ }^{1}$ Mark Ordway $^{2}$. Tim Owen ${ }^{1}$ - Jagan Ranganathan ${ }^{3}$ - Bryan A. Robertson ${ }^{1}$ • M. Janie Payne ${ }^{1}$. William Podgorski ${ }^{2}$. Jonathan Pryor ${ }^{1} \cdot$ Jenna Samra $^{2} \cdot$ Mark D. Sloan $^{14}$. Howard A. Soohoo ${ }^{1}$. D. Brandon Steele ${ }^{1}$ - Furman V. Thompson ${ }^{1}$. Gary S. Thornton ${ }^{1}$ - Benjamin Watkinson ${ }^{12}$ - David Windt ${ }^{15}$

L.A. Rachmeler laurel.rachmeler@nasa.gov

$凶$ A.R. Winebarger amy.r.winebarger@nasa.gov

1 NASA Marshall Space Flight Center, 320 Sparkman Dr, Huntsville, AL 35805, USA

2 Harvard-Smithsonian Center for Astrophysics, 60 Garden Street, Cambridge, MA 02138, USA

3 Universities Space Research Association, 320 Sparkman Dr., Huntsville, AL 35805, USA

4 College of Science, George Mason University, 4400 University Drive, Fairfax, VA 22030, USA

5 BWX Technologies, Inc., 800 Main St. Suite 400, Lynchburg, VA 24504, USA

6 Lockheed Martin Solar and Astrophysics Laboratory, 3251 Hanover st., Org. A021S, Bldg.252, Palo Alto, CA, 94304, USA

7 Rosseland Centre for Solar Physics, University of Oslo, P.O. Box 1029 Blindern, 0315, Oslo, Norway

8 Institute of Theoretical Astrophysics, University of Oslo, P.O. Box 1029 Blindern, 0315, Oslo, Norway

9 Department of Mathematics, Physics and Electrical Engineering, Northumbria University, Newcastle Upon Tyne, NE1 8ST, UK

10 Max Planck Institute for Solar System Research, 37077 Göttingen, Germany

11 Bay Area Environmental Research Institute, NASA Research Park, Moffett Field, CA 94035, USA 
Abstract The third flight of the High-Resolution Coronal Imager (Hi-C 2.1) occurred on May 29, 2018; the Sounding Rocket was launched from White Sands Missile Range in New Mexico. The instrument has been modified from its original configuration (Hi-C 1) to observe the solar corona in a passband that peaks near $172 \AA$, and uses a new, custom-built low-noise camera. The instrument targeted Active Region 12712, and captured 78 images at a cadence of $4.4 \mathrm{~s}$ (18:56:22 - 19:01:57 UT; $5 \mathrm{~min}$ and $35 \mathrm{~s}$ observing time). The image spatial resolution varies due to quasi-periodic motion blur from the rocket; sharp images contain resolved features of at least 0.47 arcsec. There are coordinated observations from multiple ground- and space-based telescopes providing an unprecedented opportunity to observe the mass and energy coupling between the chromosphere and the corona. Details of the instrument and the data set are presented in this paper.

Keywords Active regions · Corona, active $\cdot$ Chromosphere, active $\cdot$ Instrumentation and data management

\section{Introduction}

The High-resolution Coronal Imager (Hi-C) has been launched three times from White Sands Missile Range (WSMR). The first launch, Hi-C 1, occurred on July 11, 2012 (Kobayashi et al., 2014). During the 345 s of data acquisition, Hi-C 1 obtained the highest spatial resolution and highest cadence images of the extreme ultraviolet (EUV) solar corona of an active region (AR) ever achieved. Those few minutes of $193 \AA$ data have thus far generated over 25 refereed publications, including the first ever observation of coronal braiding and associated energy release (Cirtain et al., 2013). Other results include evidence of nanoflaring in magnetic (braided) coronal AR coronal loops (Winebarger et al., 2013; Thalmann, Tiwari, and Wiegelmann, 2014; Tiwari et al., 2014; Pontin et al., 2017) and in surrounding moss (Testa et al., 2013), sub-structure in coronal loops (Peter et al., 2013; Brooks et al., 2013), small-scale "sparkling" dynamic bright dots in the AR moss (Régnier et al., 2014), counter-streaming flows in filaments (Alexander et al., 2013), and moving bright dots and jets in the sunspot penumbra (Alpert et al., 2016; Tiwari et al., 2016).

Following the success of the first flight, Hi-C was modified to observe in a different wavelength to study the mass and energy coupling between the chromosphere and the corona (Hi-C 2). For this objective, the wavelength of the passband was changed to $172 \AA$ (Fe IX/X; see Sections 2.5 and 2.6, and Figure 2) and a new custom-built, low-noise camera was installed (see Section 2.3). This passband samples plasma at a relatively cool coronal or upper transition-region temperature of $\sim 1 \mathrm{MK}$. Hi-C 2 was designed to study two scientific questions: are there coronal counterparts to type II spicules; and what is the relationship between chromospheric and coronal heating in active region cores? The cooler temperature response and high temporal and spatial resolution allow the data to be combined with co-observations made by the Interface Region Imaging Spectrograph (IRIS; De Pontieu et al., 2014) to study this connection between the chromosphere and the corona.

12 University of Central Lancashire, Preston, PR1 2HE, UK

13 Space Science Division, Naval Research Laboratory, Washington, DC 20375, USA

14 Jacobs, 620 Discovery Dr., Suite 140, Huntsville, AL 35806, USA

15

Reflective X-ray Optics LLC, 425 Riverside Dr., \#16G, New York, NY 10025, USA 
The type-II spicules inject chromospheric plasma upwards at velocities of order $50-100 \mathrm{~km} \mathrm{~s}^{-1}$ and are often seen to fade as they are heated out of the chromospheric passbands (De Pontieu et al., 2007). While they may play an important role in mass and energy injected into the corona, their coronal counterparts are difficult to study because they are faint, small (diameters $<0.5$ arcsec), and short-lived (10-20 s) (De Pontieu et al., 2011). Features that may be the coronal counterparts to type-II spicules were seen in Hi-C 1 data (Régnier et al., 2014) and IRIS and SDO/AIA data (De Pontieu et al., 2017), but cospatial and co-temporal high-resolution observations of the chromosphere, transition region and corona are needed to determine the correlation.

One of the solar features targeted by Hi-C 2 is active region moss, the cool transitionregion footpoints of hot active region loops (Peres, Reale, and Golub, 1994; Berger et al., 1999; De Pontieu et al., 1999; Fletcher and De Pontieu, 1999; Martens, Kankelborg, and Berger, 2000; De Pontieu, Tarbell, and Erdélyi, 2003; Warren et al., 2008). The Hi-C 2.1 data samples $1 \mathrm{MK}$ plasma in the multi-thermal active region moss. The Hi-C 1 data showed short-lived brightenings in the moss that appeared to correlate with AIA $304 \AA$ data (Testa et al., 2013), and provide a possible signature of coronal nanoflares in the transition-region footpoints. The cooler passband of Hi-C 2, along with the co-observations, will allow for studies that correlate the brightenings in the lower corona and transition region with smallscale chromospheric dynamics in AR cores.

The Hi-C 2 payload was launched on July 27, 2016 (Hi-C 2.0). Unfortunately, an electrical short in the shutter wire prevented the camera shutter from operating and no science data were collected. Some minor changes were made subsequent to the 2016 launch, most notably the repair of the shutter cable and the addition of a Hall effect sensor to verify shutter operation pre-flight. The rocket was successfully re-launched in the summer of 2018, $\mathrm{Hi}-\mathrm{C} 2.1$. In this paper, we present the details of the Hi-C 2.1 instrument, as well as flight and data performance.

\section{Experiment Description}

The design and flight of Hi-C 1 is reviewed in detail in Kobayashi et al. (2014). For Hi-C 2.1, there were two major modifications to the published design. First, a new multilayer coating was applied to the mirrors that only reflects light near $172 \AA$; Hi-C 1 had different coating and a passband centered at $193 \AA$. Second, the original Hi-C camera was replaced by a new camera capable of significantly lower read-out noise.

In this section, we briefly describe the experiment, including the optical instrument, the camera, avionics systems, and the multilayer coatings. Much of the experiment remains unchanged from Hi-C 1, changes from the original instrument configuration are called-out specifically.

\subsection{The Experiment}

Hi-C is composed of an EUV telescope, a CCD camera, and a context telescope contained in a standard NASA 22-inch diameter rocket shell. The vehicle layout is shown in Figure 1 with all major components indicated. The EUV telescope has a plate scale of $0.129 \mathrm{arcsec} / \mathrm{pixel}-$ resolution is discussed in Section 3.2-and multilayer coatings on the full aperture of the optics. Out-of-band wavelengths are eliminated by front aperture and focal plane filters. The detector for Hi-C 2.1 is a $2 \mathrm{k} \times 2 \mathrm{k}$ back-illuminated $\mathrm{CCD}$, providing high quantum efficiency, low noise, and rapid read-out for high image cadence. An H $\alpha$ telescope with NTSC (TV) output is included for real-time pointing verification during the flight. 


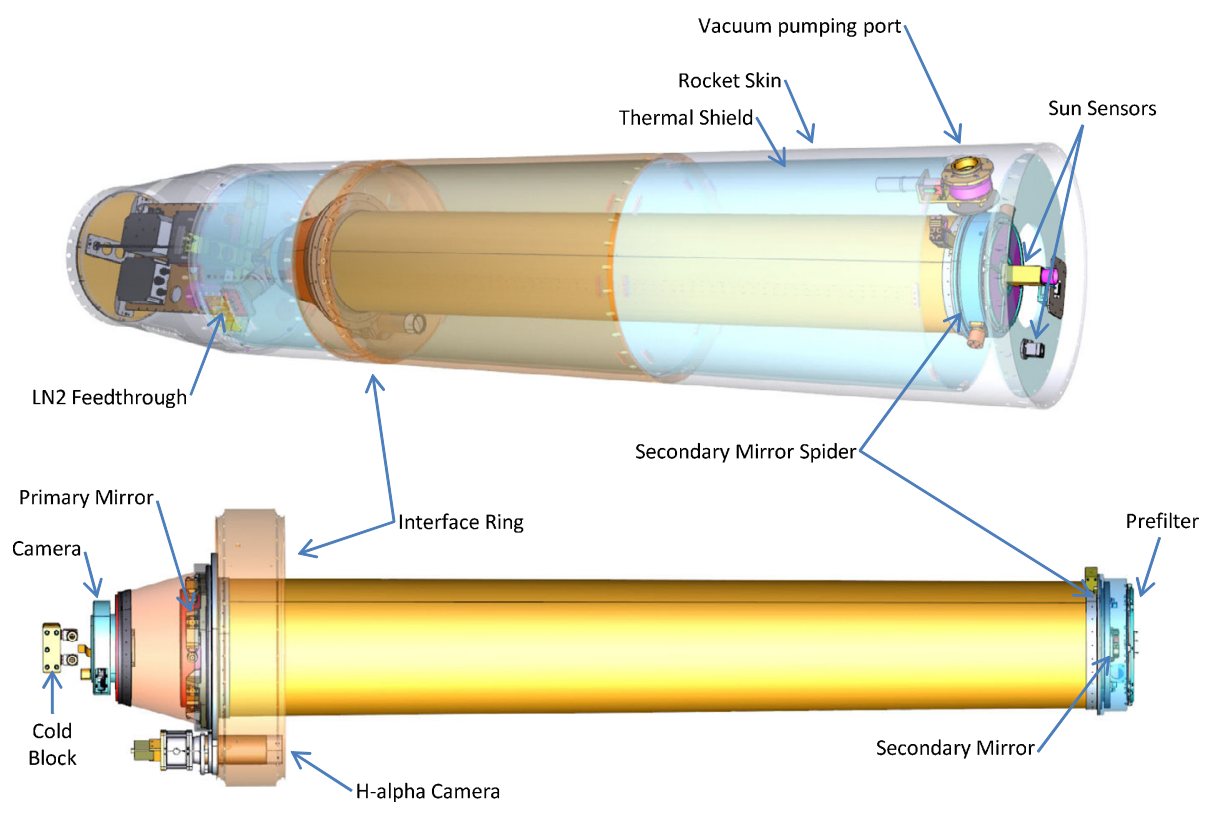

Figure 1 The Hi-C experiment layout, indicating locations of the major subsystems (Kobayashi et al., 2014).

\subsection{Telescope}

The Hi-C 1 telescope was a classical aplanatic Ritchey-Chrétien design using spherical aberration to compensate for field curvature. For $\mathrm{Hi}-\mathrm{C} 2$ the secondary was replaced with a spherical mirror with the best fit radius $(370.9 \mathrm{~mm})$ from the Hi-C 1 secondary. The telescope consists of a $220 \mathrm{~mm}$ aperture $f / 9.1$ primary mirror and a $24 \mathrm{~mm}$ (illuminated surface) secondary mirror with an inter-optic spacing of $1830 \mathrm{~mm}$, yielding an effective focal length of $23.9 \mathrm{~m}$ and providing $0.129^{\prime \prime}$ per $15 \mu \mathrm{m}$ pixel. The telescope RMS spot diameter, a baseline measure of the system resolution, is under $0.043^{\prime \prime}$ over the entire $4.4^{\prime} \times 4.4^{\prime}$ field of view (FOV). Taking into account the surface figure and roughness with the mirrors figured to the tolerances listed in Table 1, the telescope achieves $0.25^{\prime \prime}$ resolution. The surface figure was measured to $0.5 \mathrm{~nm}$ (RMS) accuracy during manufacturing to determine both mid- and high-frequency components of the mirror roughness. The performance of the telescope was initially verified against a calibrated optical flat; performance on Hi-C 1 flight confirmed that the telescope optical properties meet its required specifications (Kobayashi et al., 2014).

For the details on the original telescope mount, focus, and alignment process, performed prior to Hi-C 1 and 2 flights, see Kobayashi et al. (2014). Hi-C 2 landed at about twice the normal velocity. In order to ensure that the telescope performance was unchanged, HiC2 was returned to the Smithsonian Astrophysical Observatory (SAO) to confirm that it remained in alignment. The Hi-C telescope was placed on the SAO alignment bench, and the original telescope alignment procedure was re-run using a Zygo interferometer and a NIST-calibrated reference flat. There was a small added misalignment between the reference cube and the telescope line of sight when compared to the pre-flight alignment, but the focal plane and the imaging performance were unchanged. The slight misalignment resulted in a measurable added astigmatism, but the low spatial frequency of this defect resulted in an acceptable slope error, and thus an unchanged point spread function of the optical system. 
Table 1 Hi-C 2.1 Telescope Optical Design.

\begin{tabular}{|c|c|c|c|}
\hline \multicolumn{2}{|l|}{ Telescope: } & \multicolumn{2}{|l|}{ Primary Mirror: } \\
\hline Focal Length & $23.9 \mathrm{~m}$ & Radius of Curvature & $4000 \pm 4.0 \mathrm{~mm}$ \\
\hline Plate Scale & $114 \mu \mathrm{m} / \operatorname{arcsec}$ & Diameter & $240 \mathrm{~mm}$ \\
\hline Focal ratio & $\mathrm{F} / 109$ & RMS slope error & $0.4 \mu \mathrm{rad}$ \\
\hline Field of View & $4.4 \times 4.4 \operatorname{arcmin}$ & & \\
\hline RMS Spot Diam. & $0.08 \operatorname{arcsec}$ & Secondary Mirror: & \\
\hline (FOV averaged) & & Radius of Curvature & $370.9 \pm 0.5 \mathrm{~mm}$ \\
\hline CCD Camera: & & Conic & spherical \\
\hline Sensor Size & $942.5 \mathrm{~mm}^{2}$ & Diameter & $45 \pm 0.25 \mathrm{~mm}$ \\
\hline Plate Scale & $0.129 \mathrm{arcsec} / \mathrm{pixel}$ & RMS slope error & $0.14 \mu \mathrm{rad}$ \\
\hline
\end{tabular}

\subsection{Camera}

The camera for Hi-C 2 was custom built at MSFC for this Sounding Rocket. The primary driver for camera replacement was to reduce the noise in the images while maintaining a high-cadence. The Hi-C 1 camera noise level was between 77 and $102 \mathrm{e}^{-}$rms (Kobayashi et al., 2014). The new Hi-C 2 camera noise levels are about an order of magnitude less, between 9 and $13 \mathrm{e}^{-}$rms.

The science camera uses a CCD230-42 back-illuminated, astro-processed sensor manufactured by Teledyne e $2 \mathrm{v}$ that is operated in full-frame mode. This sensor has $2048 \times 2048$ $15 \mu \mathrm{m}$ square light-collecting pixels with 50 non-active pixels in the image read-out registers, and is operated in a read-out mode that includes two overscan pixel columns. The non-active and overscan pixels are used for image calibration. The sensor utilizes four readout registers, or taps, simultaneously with a total read-out time of $2.35 \mathrm{~s}$ for a full frame of $2152 \times 2064$ pixels. The pixels are digitized with a 16-bit resolution and the resulting images are sent to the onboard computer via spacewire.

The sensor is operated in non-inverted-mode (NIMO) to increase the read-out speed and maintain a high-cadence of science data, though it results in higher dark current at a given temperature compared to standard inverted mode (IMO). The CCD is cooled via liquid nitrogen (LN2) to maintain a temperature below $-65{ }^{\circ} \mathrm{C}$ during observations to minimize the higher dark current. LN2 is pumped into the payload and through a copper cold block that is connected to the copper CCD holder via a cold strap. The cold block rose from $-122.2^{\circ} \mathrm{C}$ to $-117.0^{\circ} \mathrm{C}$ during observations. The cold block is significantly colder than the $\mathrm{CCD}$, the $\mathrm{CCD}$ temperature dropped from $-78.4{ }^{\circ} \mathrm{C}$ to $-79.1^{\circ} \mathrm{C}$ during observations. During lab testing we determined that the dark current reached a floor of $\sim 3$ electrons/s when the sensor was below $-65^{\circ} \mathrm{C}$; the $\mathrm{CCD}$ was below this floor during all of the data acquisition during flight.

The full-well depth of the sensor is $\sim 150,000$ elec, with a 16-bit resolution. A gain value of $\sim 2.5$ elec $\mathrm{DN}^{-1}$ was chosen so that digital saturation was commensurate with full well saturation. This allowed us to maximize the dynamic range of the sensor. This gain value was confirmed in laboratory testing.

Camera characterization in the laboratory included: dark current as a function of the temperature, read noise, bias, linearity and saturation, and gain measurements. Each tap has slightly different characteristics, which are described in Section 4 and summarized in Table 4. 


\subsection{Electronics}

Hi-C's avionics consist of a Data Acquisition and Control System (DACS), low-noise power supply, shutter drive, signal conditioning vacuum valve controller, and two vacuum gauges.

The DACS controls camera operations and performs data collection, processing, and transmission; it is an $\times 86$ architecture computer running Linux Fedora 19. The hardware consists of a CompactPCI backplane, a conduction-cooled Single-Board Computer, SBC, (AiTech C802), Spacewire card, digital I/O board, and 500 GByte solid-state storage hard disk drive, and a power supply; these components are packaged in a custom-designed chassis. The digital I/O board, custom designed at MSFC, contains a 16-bit parallel interface that is used to send formatted image data to the $10 \mathrm{Mbps}$ telemetry system. The Spacewire card interfaces to the science camera.

The Hi-C DACS has three interfaces to the telemetry system. The 16-bit parallel interface on the digital I/O board, custom designed at MSFC, is used for downlink of acquired science images. A serial uplink channel is used for command uplink, and a serial downlink channel is used for digital housekeeping and status data. Additional telemetry channels are used for analog housekeeping data (temperatures, power supply voltages, supply currents, and camera shutter drive current) via the signal conditioning, and for the video signal of the $\mathrm{H} \alpha$ camera.

While uplink commands are available, they are only used for redundancy; the DACS flight software is designed to operate without need for uplink commands. Instead, timer commands from the rocket's timer system are used to initiate changes in operating mode: standby, dark frame acquisition, observation, and shutdown. Additional uplink commands allow for exposure time changes if the downlinked images show saturation or severe underexposure. No uplink commands were sent during the Hi-C 2.1 flight.

The DACS and flight camera are capable of sustained 4.4 s cadence with a 2.0 s exposure time. Every image that is taken is saved on a solid-state onboard disk in full-resolution as individual files in standard FITS format. A limited amount of data can be downlinked to the ground during flight to aid in pointing corrections and to preserve a subset of the data for scientific use in the event that the onboard storage is damaged during flight or landing. The downlinked images used for pointing corrections were resampled to a lower $1026 \times$ 1032 spatial resolution as higher cadence is needed for real-time pointing and exposure corrections and downlink speed is limited. The scientific goals of the mission require fullresolution data so after the pointing phase, some of the full $2152 \times 2064$ pixel resolution images are downlinked. Due to the limited downlink bandwidth, only a subset of the fullresolution images can be downlinked during flight.

A requirement unique to $\mathrm{Hi}-\mathrm{C}$ was to monitor the internal vacuum and operate the vacuum valve during the flight, as well as during the countdown phase before the DACS is powered on. The vacuum valve is opened just before the main telescope door, to equalize pressure and minimize the chance of the thin front aperture filter being damaged by a sudden rush of air due to a pressure imbalance when the main telescope door is opened. The DACS is turned on only 10 min before flight with the rest of the telemetry system. Separate power is provided via a dedicated payload umbilical and used to operate the vacuum valve, monitor instrument vacuum, and cool the camera CCD to flight operating temps without needing the DACS or telemetry subsystems. This requirement was addressed by using RS485 interfaces that can be put into a tri-state mode; both the DACS and the Ground Support Equipment (GSE) computer can take control of the vacuum valve controller and vacuum gauge. External GSE is used to control cooling of the science camera CCD.

The low-noise power supply provides eight secondary voltages isolated from the primary 28 Volt input for the science camera and supporting electronics. The power supply has low 

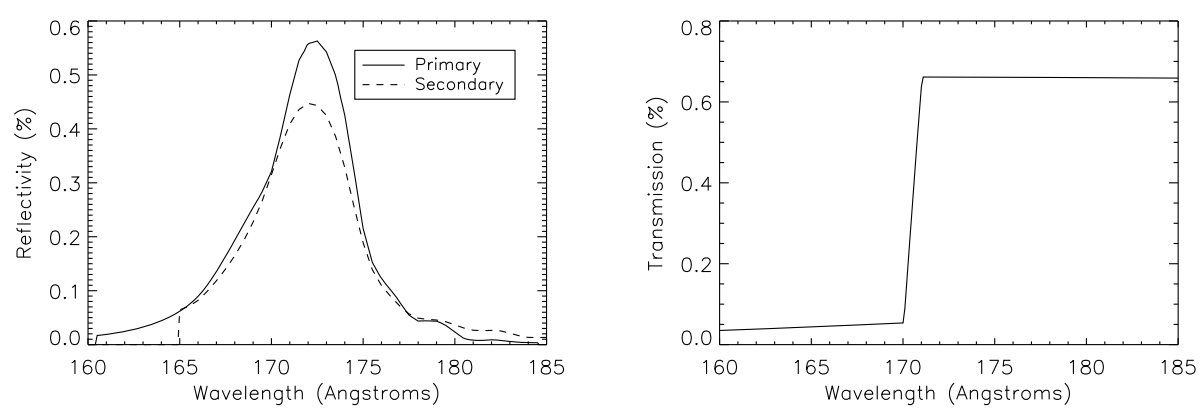

Figure 2 Left: The reflectance of the multilayer coating measured from a witness sample of the primary (solid) and secondary mirror (dashed). Right: Theoretical transmission of the individual entrance and focal plane filters.

ripple and switching noise to enable the low read noise requirements for the science camera. The power supply also provides opto-isolated monitor outputs to telemetry for voltages and currents.

\subsection{Multilayer Coatings}

As with most solar EUV telescopes, the passband is selected through a combination of a filter that blocks most of the solar light and heat from entering the telescope, and a multilayer coating on the optical surfaces that only reflects a narrow range of wavelengths. A new coating was used for Hi-C 2. The Hi-C 1 primary mirror was removed from the telescope, cleaned, and re-used for Hi-C 2 . The secondary mirror was new for Hi-C 2 . The mirrors were coated with periodic $\mathrm{Al} / \mathrm{Zr}$ multilayers designed for high reflectance near normal incidence over a narrow spectral band centered near $17.2 \mathrm{~nm}$ wavelength. Multilayer interference coatings comprising $\mathrm{Al}$ and $\mathrm{Zr}$ layers have been demonstrated in recent years to provide high reflectance at wavelengths longer than the $\mathrm{Al} \mathrm{L}$-edge near $17 \mathrm{~nm}$, where photoabsorption in the Al layers is relatively low (Voronov et al., 2011). Al/Zr multilayer coatings also have been shown to have good stability and low stress. For the aluminum layers, an Al-Si alloy containing $1 \% \mathrm{Si}$ (by weight) can be used in place of pure $\mathrm{Al}$ in order to achieve smoother $\mathrm{Al}-\mathrm{Zr}$ interfaces and thus higher reflectance (Zhong et al., 2012; Windt, 2015). The coatings designed for Hi-C 2 thus contain $N=20$ repetitions of Al.99Si.01-Zr bilayers of thickness $d=8.75 \mathrm{~nm}(d \mathrm{Al}-\mathrm{Si}=5.25 \mathrm{~nm}$ and $d \mathrm{Zr}=3.5 \mathrm{~nm})$. The coatings were deposited by magnetron sputtering, using a deposition system described in Windt and Waskiewicz (1994). Sputter target purities were $99.95 \%$ for $\mathrm{Zr}$ and $99.999 \%$ for Al.99Si.01. DC power supplies were used in constant-power mode $(400 \mathrm{~W})$ for both materials. The deposition system achieved a base pressure of $1.5 \times 10^{-7}$ Torr after pump-down, and $\operatorname{Ar}(99.999 \%$ purity) was used as the working gas, fixed during deposition at a pressure of 1.6 mTorr using a closedloop gas-flow system. Si witness samples were coated along with the telescope mirrors. The EUV reflectance of the coated mirrors was measured as a function of wavelength, at $2^{\circ}$ incidence, using a reflectometer system with a laser-produced-plasma source, also described in Windt and Waskiewicz (1994). The measured reflectance as a function of wavelength of a witness sample is shown in Figure 2, showing peak reflectance of 55\% and a spectral band-width of $0.4 \mathrm{~nm}$ FWHM. 


\subsection{Filters}

The Hi-C experiment included two thin-film filters, one at the entrance to the telescope and one in the focal plane. The filters are always new for each flight as they are easily damaged due to their fragility, they are tuned to transmit only specific wavelengths, and they oxidize quickly. Both filters were $150 \mathrm{~nm}$ of aluminum film mounting on five lines per inch nickel mesh in custom-designed frames made by Luxel. The material is identical to the entrance and focal plane filters of the Solar Dynamics Observatory Atmospheric Imaging Assembly (SDO/AIA) $171 \AA$ channel (Lemen et al., 2012), but the AIA filters have a much finer mesh. Transmission curves for the filters are shown in the right hand side of Figure 2. When calculating the transmission of the filters, we assume that the mesh has $98 \%$ area.

As with Hi-C 1, the focal plane filter was situated close enough to the telescope focus to create a mesh shadow-pattern on the images. The removal of this grid pattern in postprocessing is described in Section 4.

\subsection{H $\alpha$ Camera and Pointing GUI}

The primary function of the H $\alpha$ camera system is to confirm that the telescope is pointing at the selected target and to provide context for gross pointing maneuvers in the case of significant pointing error. (The EUV images from the science camera are used for fine pointing correction.) The $\mathrm{H} \alpha$ camera is composed of a $712 \mathrm{~mm}$ effective focal length negative telephoto lens system, a Day-Star Corporation H $\alpha$ narrow band-pass $(0.6 \AA)$ filter centered at $6563.28 \AA$, neutral density filters, and a Sony monochrome camera. This system is identical to the one flown on the Tunable XUV Image (TXI) rocket, which produced a full Sun image with good contrast and resolution, allowing us to recognize sunspots, and confirm pointing orientation.

To improve the functionality of the $\mathrm{H} \alpha$ camera system for Hi-C 2.1, a graphical user interface (GUI) application was developed to use the $\mathrm{H} \alpha$ images to determine, in real-time, the pointing of the telescope relative to the pre-determined target. An image processing algorithm was developed by the University of Central Lancashire (UCLAN) to locate the Sun center within the $\mathrm{H} \alpha$ image, which was then cropped to the edge of the determined solar limb. Based upon where the Sun center is located in this image and the offset between the $\mathrm{H} \alpha$ camera and science camera FOV (measured pre-flight), a box was outlined in real-time on the H $\alpha$ image to display the current pointing of the EUV camera. A second box was also displayed for the pre-planned FOV in order to determine any differences in telescope pointing (Figure 3). Thus, this information allowed for any significant pointing differences to be recognized immediately and a decision made on any need for subsequent adjustment. During the flight, H $\alpha$ data were captured at a rate of one in every ten frames and saved as FITS files for further post-flight analysis. A similar application is being developed by UCLAN to aid in pointing of the Marshall Grazing Incidence X-ray Spectrometer (MaGIXS) instrument using data from the MaGIXS slit jaw camera.

\subsection{Radiometry and Exposure Time Estimate}

Before flight, it was important to calculate the expected throughput of the instrument and predict an adequate exposure time to prevent saturation or under-exposure. Using the geometric area of the telescope, the measured reflectance curves of the multilayers (Figure 2, left panel), transmission of the entrance and focal plane filters (Figure 2, right panel), and the expected quantum efficiency of the CCD, we have calculated the effective area of the 
Figure 3 Image from the real-time $\mathrm{H} \alpha$ pointing GUI during on-ground testing. The blue box shows the intended target and the green box shows the current target.
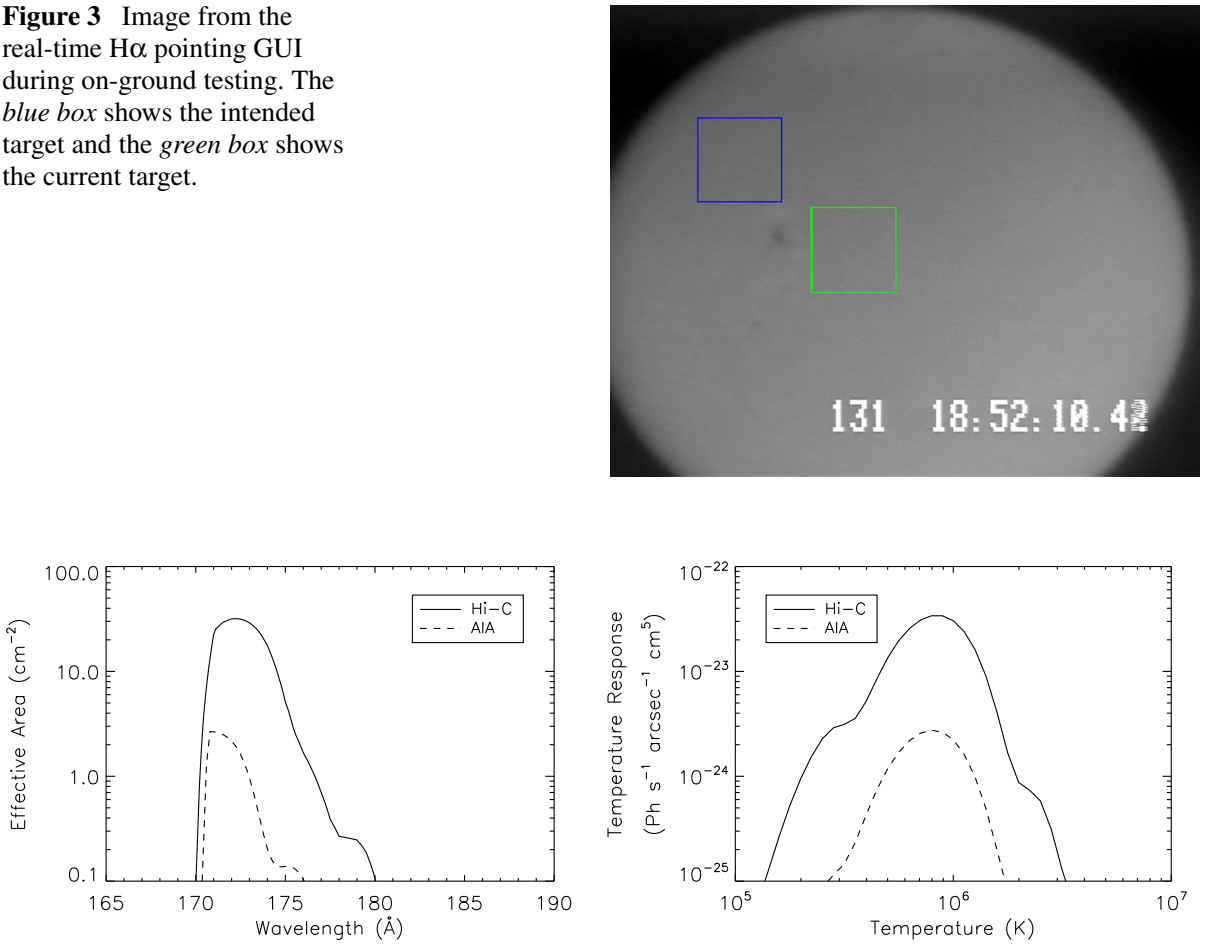

Figure 4 The effective area (left panel) and temperature response (right panel) of the Hi-C $2.1172 \AA$ passband (solid) and AIA $171 \AA$ channel (dashed).

Hi-C $2172 \AA$ passband; this is shown in Figure 4. For comparison, we show the effective area of the AIA $171 \AA$ channel with a dashed line, which was calculated using the options of EVE normalization and the time-dependent correction for the Hi-C 2.1 launch date. The effective area of the Hi-C $2.1172 \AA$ passband is broader in shape, shifted in wavelength (peak is at $170.8 \AA$ in AIA and at $172 \AA$ in Hi-C 2.1), and 11.9 times larger than the AIA $171 \AA$ channel. Using these two effective area curves, we calculate the temperature response for both Hi-C and AIA assuming standard Chianti ionization equilibrium and coronal abundances from Schmelz et al. (2012). These curves are shown on the right side of Figure 4. The Hi-C $2.1172 \AA$ and AIA $171 \AA$ temperature responses both peak at $\log T=5.9$.

Hi-C 2.1 was expected to transmit 10.7 times more signal per arcsec than the AIA $171 \AA$ channel, but it has roughly 22 pixels for each AIA pixel. Based on this calculation and our previous experience with the first flight of Hi-C (Winebarger et al., 2014), we estimated that a $2 \mathrm{~s}$ exposure time was appropriate for this flight.

After flight, we used co-temporal AIA data to validate the radiometry of Hi-C 2.1. Because the shape of the Hi-C 2.1 passband was slightly different from the AIA $171 \AA$ channel, we use the six AIA EUV channels taken closest to the time that Hi-C 2.1 was at apogee (where Hi-C 2.1 data suffers least from atmospheric absorption; see Figure 7) and calculate an emission measure curve at every AIA pixel using the method of Cheung et al. (2015), this emission measure distribution is shown in Figure 5. We then convolve the emission measure with the Hi-C 2.1 temperature response function and predict the expected counts in Hi-C 2.1. We find that the counts in $\mathrm{Hi}-\mathrm{C}$ are $15 \%$ larger than predicted from this method. We assume 


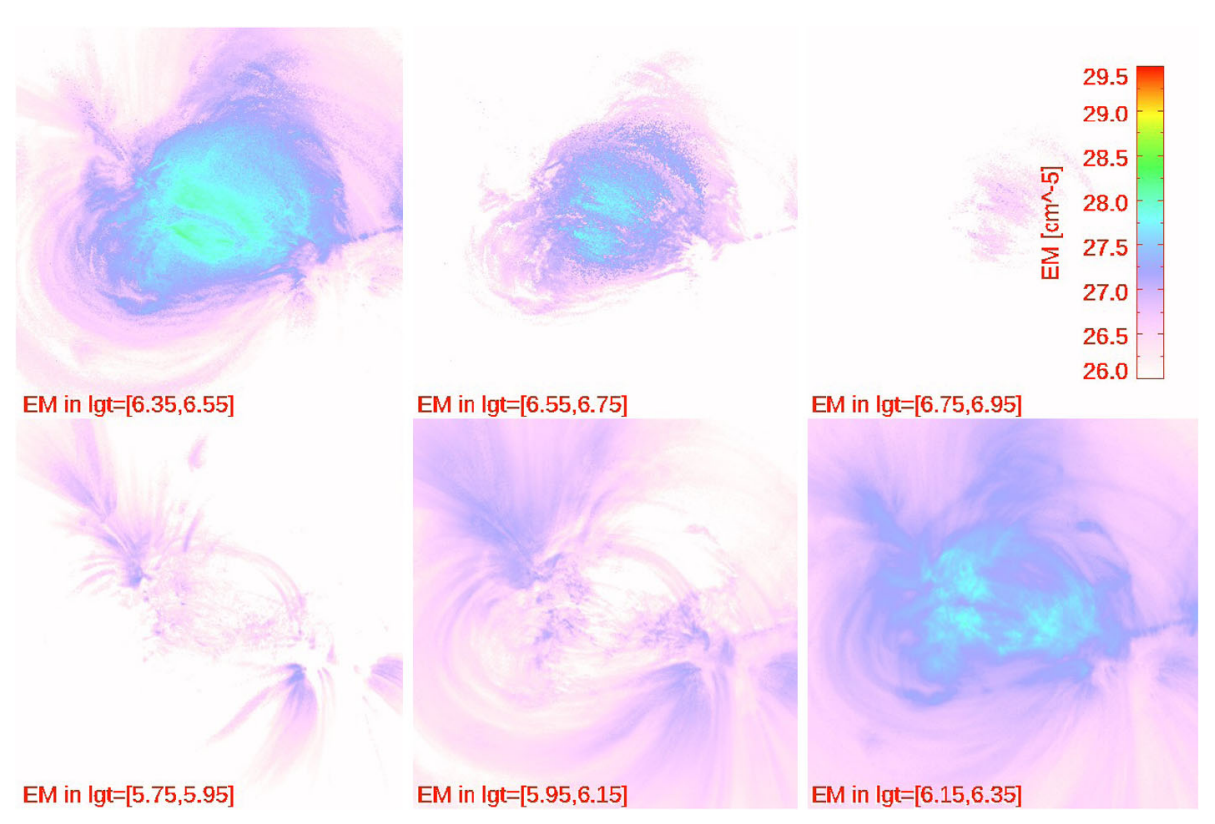

Figure 5 The emission measure distribution calculated from co-temporal and co-spatial AIA data.

Table 2 Hi-C 2.1 flight event timeline (May 29, 2018)

\begin{tabular}{lll}
\hline & Event & Time (UTC) \\
\hline 0 & Launch & $18: 54: 00.41$ \\
1 & Start Dark Exposures & $18: 54: 04$ \\
2 & End Dark Exposures & $18: 55: 06$ \\
3 & Shutter Door Open & $18: 55: 12$ \\
4 & Fine Pointing [Ring Laser & $18: 56: 01$ \\
& Gyroscope (RLG) Enable] & $18: 56: 21$ \\
6 & Data Acquisition & $19: 02: 00$ \\
\hline
\end{tabular}

this $15 \%$ is within the uncertainty of the AIA calibration and the Hi-C component level effective area calculation.

\section{Flight Performance}

Hi-C 2.1 was launched at 18:54 UT on May 29, 2018 from White Sands Missile Range. The target of observation was Active Region 12712. The Solar Pointing and Aerobee Control System (SPARCS) maintained a constant target for the duration of the flight. For $335 \mathrm{~s}$, HiC 2.1 recorded full detector $(\sim 2 \mathrm{k} \times 2 \mathrm{k})$ images with a $2 \mathrm{~s}$ exposure at a cadence of $4.4 \mathrm{~s}$. Because the time on the Hi-C onboard DACS drifts, an adjustment of $126 \mathrm{~s}$ was applied to all data headers in post-processing. Table 2 provides the timeline of the Hi-C 2.1 rocket flight. Figure 6 provides the height of the sounding rocket as a function of time, determined 
Figure 6 The altitude of the Hi-C 2.1 rocket determined from White Sands Missile Range radar data as a function of elapsed time from launch (18:54:00 UT). The event times listed in Table 2 are labeled.

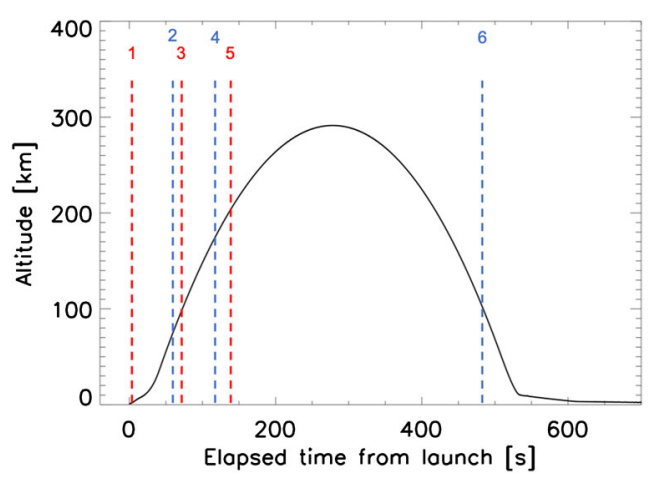

Figure 7 The relative transmission of the Hi-C 2.1 data (plus symbols) and payload altitude (solid line) as a function of time elapsed since launch (18:54:00 UT).

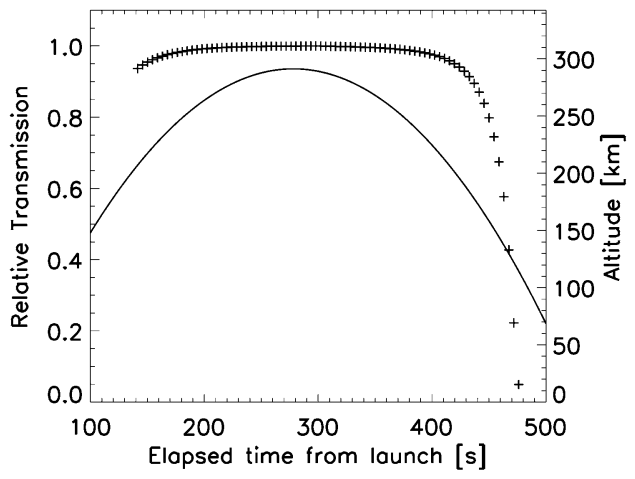

from White Sands Missile Range radar measurements. The events given in Table 2 and the approximate height at which they occurred are indicated in this figure.

We use the normalized total intensities of the Level 1.0 processed flight data (processing levels described in Section 4) to assess the relative atmospheric absorption of the signal as a function of flight time. The transmission, shown in Figure 7 in combination with the payload altitude, is calculated as the inverse of the relative absorption (i.e., (absorption coefficient $)^{-1}$ ). More than 4 minutes of data were unaffected by the atmosphere. The atmospheric absorption was compensated for in the Level 1.5 processed data set by multiplying the images by their respective absorption coefficient. These coefficients are provided in the header of this processed set.

\subsection{Pointing}

As with the Hi-C 1 flight, the $\mathrm{H} \alpha$ pointing camera system described in Section 2.7 did not take on-band images. The wavelength is controlled by a temperature-sensitive etalon, and the heating caused by exposure to full sunlight under vacuum caused a wavelength shift. The full-sun continuum images were sufficient to verify coarse pointing with the GUI system described in Section 2.7, which was further refined using downlinked the science camera images.

To determine the roll offset and absolute pointing post flight, the AIA $171 \AA$ image taken at 18:56:57.35 UT was used as a reference against the Hi-C2.1 image taken at 18:56:56.64 UT. The roll offset, found to be $\sim 0.985 \pm 0.005^{\circ}$ (clockwise about Sun center), is within the tolerances for SPARCS pointing. Figure 8 shows the full-disk AIA $171 \AA$ 
Figure 8 The reference AIA

$171 \AA$ data taken at 18:56:57 UT, which was used for determining the absolute pointing. The highlighted box indicates the Hi-C 2.1 FOV.

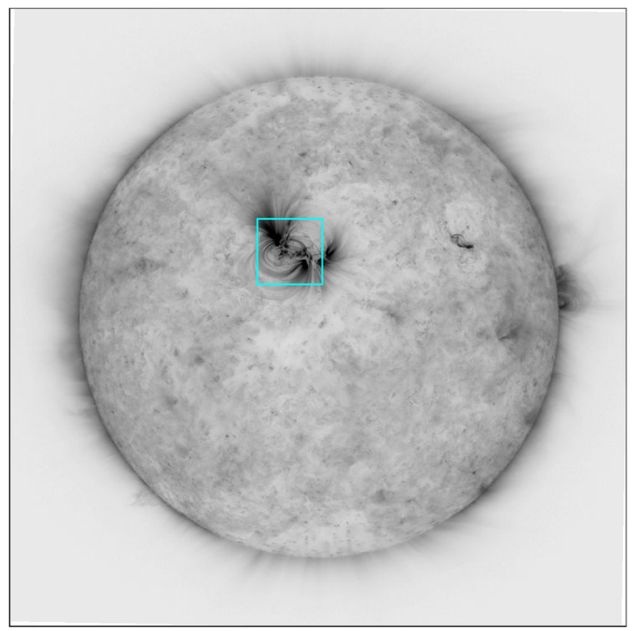

image rotated to this offset. The Hi-C $2.1 \mathrm{FOV}$, centered at $\left(-114^{\prime \prime}, 259^{\prime \prime}\right)$, is indicated by the box.

The full Hi-C 2.1 image set was co-aligned through a combination of cross-correlation techniques. The target region drifted in the FOV by $\sim 3$ pixels due to solar rotation during the flight, as determined by an AIA image set spanning the flight. The solar rotation rate at a latitude halfway through the FOV $\left(15^{\circ}\right)$ is $\sim 14.5^{\circ}$ day $^{-1}$, which corresponds to $\sim 0.45^{\prime \prime} \mathrm{drift}$ at a longitude of $-15^{\circ}$ as observed from Earth over the course of $326 \mathrm{~s}$ of observations. This calculated drift corresponds to $\sim 3.5 \mathrm{Hi}-\mathrm{C}$ pixels, which is consistent with the measured $3 \pm 1$ pixel drift. We use the measured value to approximately correct for the drift in the Level 1.5 data set. The headers of the Level 0.5 and higher data files were adjusted to include the best approximation of the absolute pointing for each image, including the fine shifts.

\subsection{Stability and Resolution}

Though Hi-C 2.1 achieved all pointing and stability requirements, enabling the acquisition of the highest resolution $17.2 \mathrm{~nm}$ coronal images ever taken, approximately half of the images captured during flight show signs of greater than expected motion blur. The stability requirements were RMS pitch and yaw jitter of $<0.3^{\prime \prime}$ and RMS roll jitter of $<0.01^{\circ}$ for $90 \%$ of the observation time. These requirements were met as shown by recorded jitter as a function of time (Figure 9). The measured RMS jitter values were $\sigma_{\text {yaw }}=0.06^{\prime \prime}, \sigma_{\text {pitch }}=0.05^{\prime \prime}$ and $\sigma_{\text {roll }}=0.0014^{\circ}$. Some exposures indicate pointing instability resulting in motion blur and lower spatial resolution. This motion blur is observed to be semi-periodic, impacting every sixth-eighth frame most severely. The most likely source of motion blur is roll instability, a semi-periodic variation can be seen in the measured roll data (Figure 9C).

The image resolution is a function of both the instrument PSF and the motion blur. At best, the image resolution must be greater than or equal to $0.26^{\prime \prime}$, twice the pixel plate scale. In Hi-C 2.1 the resolution changes with time, implying that motion blur was acting to degrade the resolution of some of the images. The spatial resolution has been approximated by Fourier analysis (Figure 10), and by analyzing intensity cross sections of fine structures (Figure 11).

A 2D Fourier transform describes the spatial frequency information contained in an image (Young, Gerbrands, and van Vliet, 2011). By assessing the spatial frequency content of 


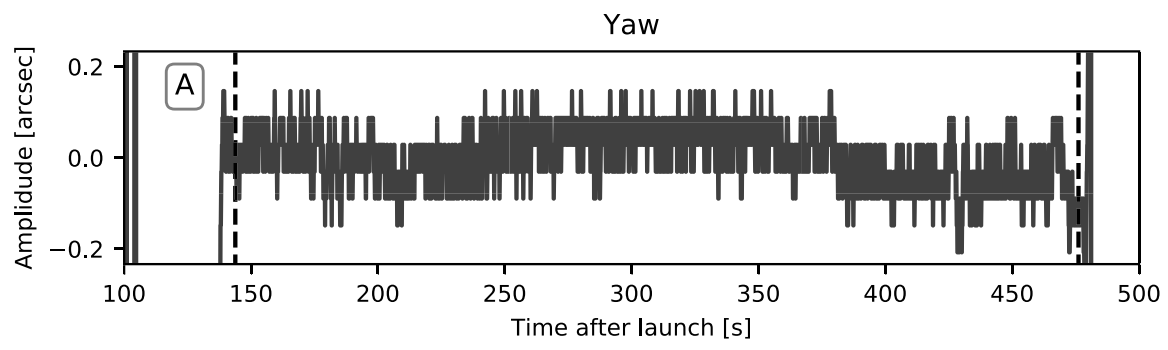

Pitch

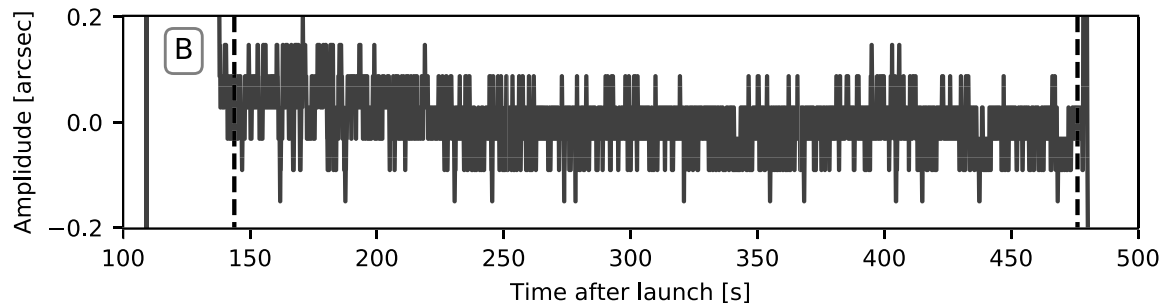

Roll

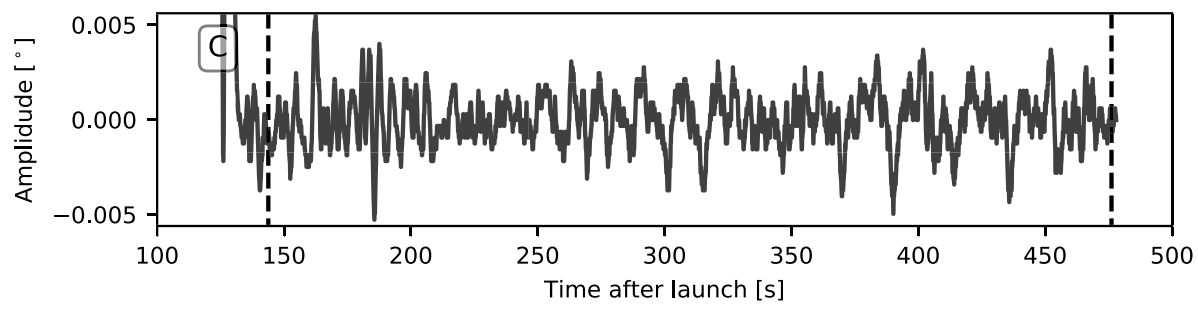

Figure 9 Pointing stability as a function of time during the imaging period. Yaw (A), pitch (B) and roll $(\mathbf{C})$ are plotted for the entire imaging period. Image acquisition period is indicated by dashed black lines. Notice a semi-periodic variation of roll pointing during observation.

an image, relative resolution performance of individual frames can be compared and a rough estimate of the image resolution obtained. A similar analysis was applied in Kobayashi et al. (2014) for Hi-C 1 data. In Hi-C 1, the frequency spectrum followed the slope of AIA $171 \AA$ frequencies down to at least $3-4 \operatorname{arcsec}^{-1}$ when an average of four sequential Hi-C 1 frames was used to reduce the noise. Due to time-varying nature of the Hi-C 2.1 images, no four subsequent frames maintain a consistent resolution, and this method is not as applicable. However, we can still analyze the Fourier information to determine the spatial scale of the noise-floor of a single image.

Figure 10 shows two sub-frames from the Hi-C 2.1 data set, one that experienced low jitter (A) and one that was significantly blurred (B). The corresponding field of view acquired in the AIA $171 \AA$ channel is shown (C) for comparison. The azimuthally averaged full-frame frequency power spectrum of the Hi-C 2.1 frames and AIA $171 \AA$ A sub-image are plotted (D). The major and minor axis of width (i.e. extent) of the 2D fast Fourier transform (FFT) as a function of time is shown in (E). The widths are approximated by the inflection point where the spectra becomes dominated by a function differing from that which defines the lower frequency components. The FFT width varies between $\approx 0.35-1.1^{\prime \prime}$ and oscillates between sharp and blurred with a period of approximately six-eight images, or 27-36 s. 

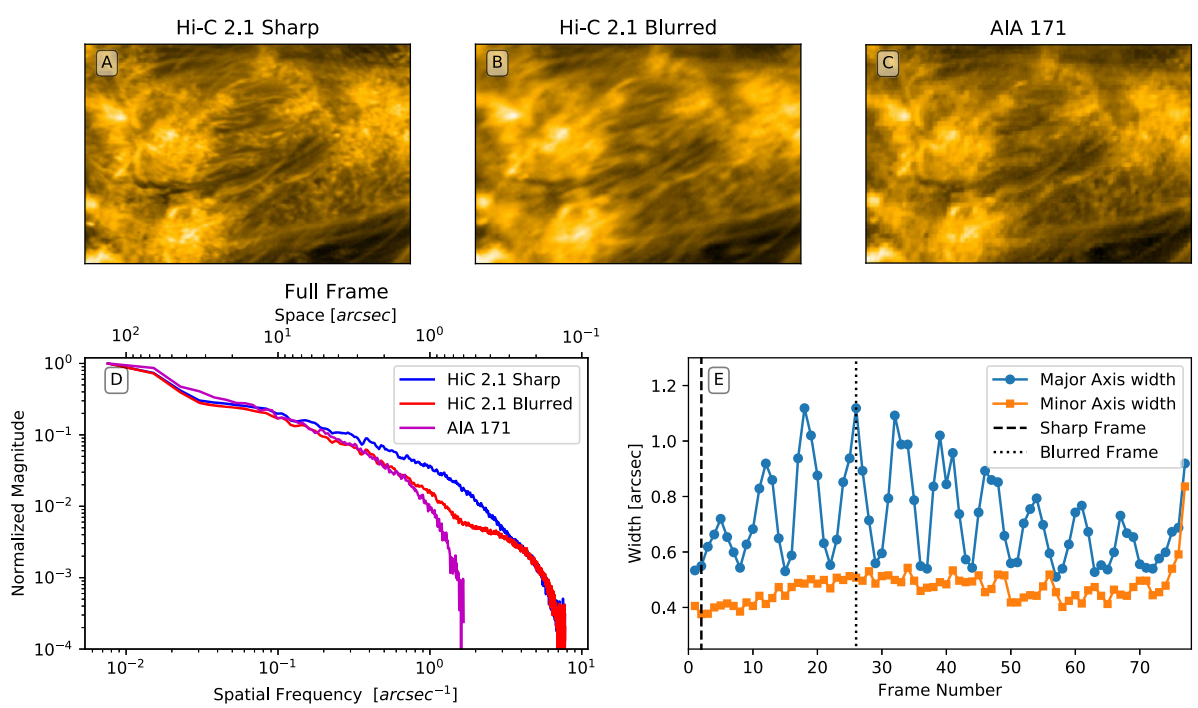

Figure 10 A sharp image (A) and a motion blurred image from the Hi-C 2.1 data set (B) are given with the corresponding AIA 171 sub-image (C). The full-frame averaged FFT (D) plotted with space (top axis) and spatial frequency (bottom axis). The major and minor axis of the FFT width is shown in (E). The vertical lines correspond to the frame numbers of the example sharp (dashed) and blurred (dotted) images.

The curvature of the FFT (Figure 10D) shows that the blurred image (red line) has a clear inflection point at approximately $0.6^{\prime \prime}$, indicating that below this level, the image is being impacted by motion blur. The lack of a clear inflection point for the sharp image (blue line) indicates the image is limited by instrument resolution or scale of structures in the image instead of by motion blur. The sharp image FFT deviates from the AIA 171 A FFT (magenta) at spatial scales of approximately $5^{\prime \prime}$, while the blurred image FFT (red) deviates from AIA at around 1.5". These deviations signify the spatial scales at which Hi-C data are better resolved than AIA. Further, both the sharp and the blurred Hi-C curves converge again around $0.3^{\prime \prime}$, indicating that features below this scale are not well resolved in any single HiC 2.1 image, being limited by shot noise and nearing the instrument Nyquist frequency.

The Fourier spectrum is related to the image resolution, but not exactly equivalent. The spectrum will change based on the spatial frequency content present in the objects being imaged, and so this analysis is dependent on the target selection. The Fourier analysis does clearly show that the Hi-C 2.1 images have a clear periodic resolution degradation due to motion blur, and it gives a limit to spatial scales resolved in the images but does not provide an unambiguous measurement of image resolution.

In assessing Hi-C 2.1 resolution performance, it is also helpful to examine directly the widths of small features in un-blurred frames. Gaussian fits corresponding to a selection of small features are listed in Table 3. The locations for each feature are noted in Figure 11 along with an example of the cross section and fitting performed. Of these selected features, the smallest resolved Gaussian width is $\approx 0.47^{\prime \prime}$, implying that the image resolution is better than $0.47^{\prime \prime}$. True resolution can be assessed from the imaged width of a sub-resolution feature such as a point source. However, the features summarized in this list do not represent sub-resolution features, and therefore can only provide an upper bound on resolution. These features were manually selected from a single frame and this analysis does not rule out the possibility of resolved smaller features, especially if they are present in multiple frames. 
Table 3 Feature cross section list. Feature locations are shown in Figure 11. Listed location is the pixel index of beginning and end of the cross sectional line, where the first pixel in the image is $[0,0]$.

\begin{tabular}{llll}
\hline Label & Location & FWHM $_{1}\left({ }^{\prime \prime}\right)$ & FWHM $_{2}\left({ }^{\prime \prime}\right)$ \\
\hline A & {$[1027,1133],[847,963]$} & 0.7 & 0.66 \\
B & {$[887,989],[602,719]$} & 0.94 & \\
C & {$[521,644],[833,933]$} & 1.61 & \\
D & {$[850,955],[1375,1520]$} & 2.95 & 1.2 \\
E & {$[1368,1469],[962,1075]$} & 0.83 & \\
F & {$[780,906],[1436,1546]$} & 2.11 & 0.98 \\
G & {$[1804,1919],[978,1081]$} & 0.89 & \\
H & {$[19,127],[1337,1462]$} & 1.07 & 0.90 \\
I & {$[1559,1670],[1398,1520]$} & 0.88 & 1.44 \\
J & {$[206,310],[1582,1690]$} & 0.83 & \\
K & {$[944,1050],[1008,1125]$} & 0.47 & 1.07 \\
L & {$[1717,1834],[1545,1652]$} & 1.1 & \\
\hline
\end{tabular}

Combining both FFT and Gaussian width analysis methods, we conclude that the $\mathrm{Hi}-\mathrm{C} 2.1$ resolution is between 0.3 and 0.47 arcsec in images that are not affected by motion blur.

\section{Data}

The Hi-C 2.1 images are processed by subtracting the bias read-out pedestal and the dark current, flat-fielding, and correcting for bad pixels.

Bias The non-active regions of the images (see Section 2.3) are used to determine the bias pedestal, which varies slightly between quadrants and as a function of time. The bias is calculated and removed per frame and per quadrant.

Dark Current During ascent, 15 dark frames were obtained matching the exposure time of the science images (i.e., the images exposed to sunlight). A master dark frame was created from a median of these images (to remove the contribution of particle hits), after removal of the bias in each of the individual dark frames. This master dark (Figure 12A) is also subtracted from all of the science images.

Flat-Field The data were further affected by the shadow of the mesh from the focal plane filter, reducing the intensity behind the mesh by up to $\sim 35 \%$. The mesh pattern and its associated transparency was derived from the blurred, exposed images taken while slewing during the initial fine pointing procedures. A master flat-field image was created to compensate for the mesh only, it does not affect the unobscured pixels. The data were divided by this master flat (Figure 12B) to reduce the presence of the grid.

Note that due to the lack of a flat EUV source for measuring the flatness of the detector on the ground within a sounding rocket flight budget, it is not possible to determine the true intensity variability across the field of view; however, visual inspection of the images indicates that any variability between the mesh grid is likely insignificant. Likewise, the error in the intensity reduction of the mesh cannot be precisely determined from the small number of blurred, exposed images used to create the flat-field. This flat-field is intended primarily as an aesthetic correction and is made available with the data for users to reference the affected pixels. 

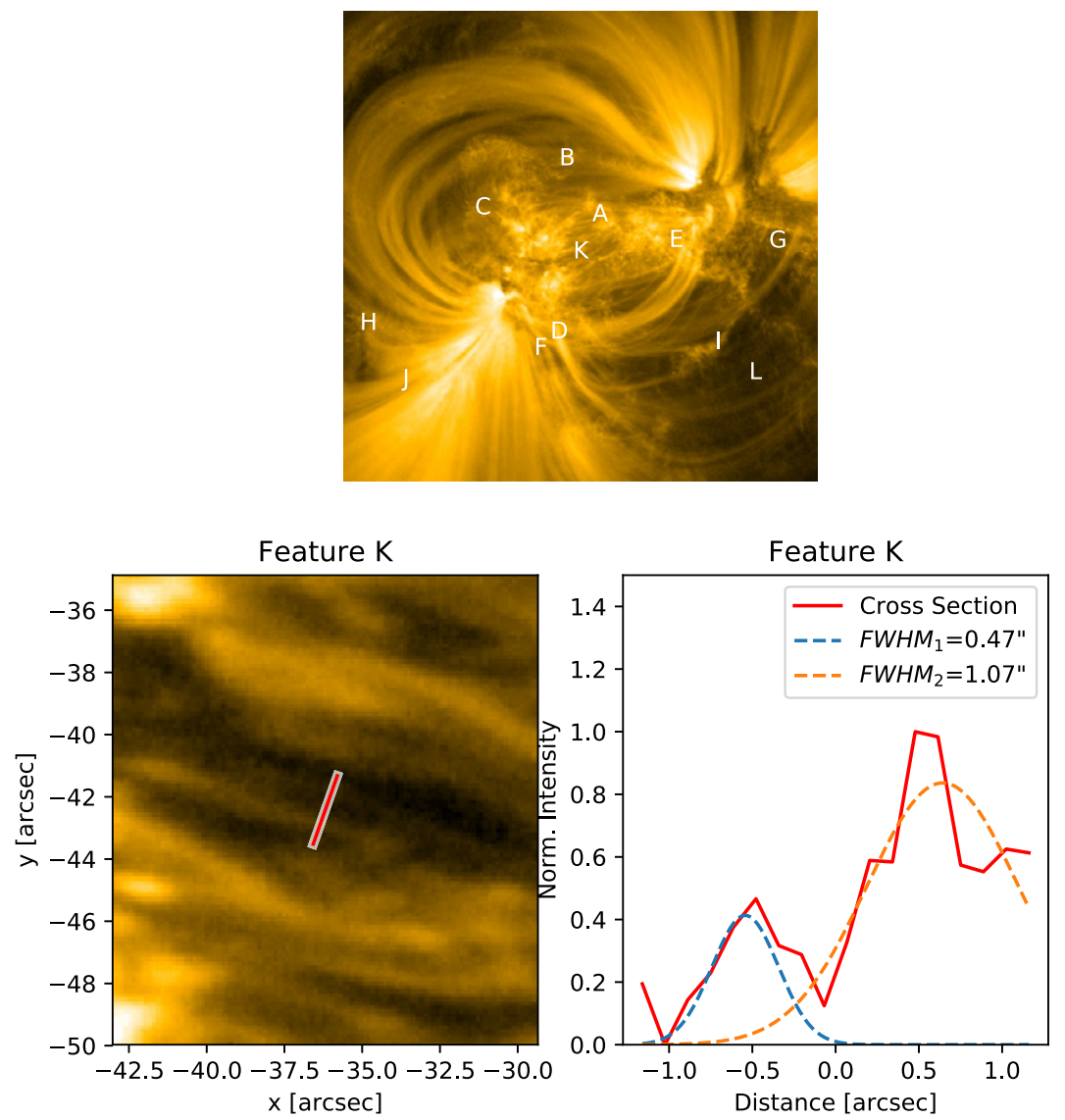

Figure 11 Feature cross sections and fitted widths. Sharp frame of Hi-C 2.1 data set marked with locations of features sampled (top) which correspond to Table 3. A zoomed-in example of a sample feature (bottom left) with intensity along the cross section and Gaussian fits (bottom right). FWHM of the Gaussian fits are given in the legend.

Bad Pixels Finally, a bad pixel map (including pixels obscured by dust, Figure 12C) was generated by applying a threshold to the dark frames and the science images that were blurred during initial fine pointing procedures. The adjusted intensity in the affected pixels is interpolated from the nearest pixels that are not identified as containing dust or bad pixels.

Raw and calibrated images are shown in Figure 12D and E. (Note that the raw image has been rotated by $90^{\circ}$ counter-clockwise prior to processing, in order to place solar north toward the top of the frame, and the non-active pixels have been cropped.)

Gain The camera gain was determined pre-flight (using an Fe55 source) to be 2.5 electrons $\left(\mathrm{e}^{-}\right)$per data number $(\mathrm{DN})$, with variability less than 0.02 elec $\mathrm{DN}^{-1}$ between quadrants.

Noise The camera was cooled below the required $-65^{\circ} \mathrm{C}$ during flight and therefore achieved low dark current. The remaining noise is dominated by the camera read noise. The noise in Table 4 is calculated as the median standard deviation per quadrant of the set 

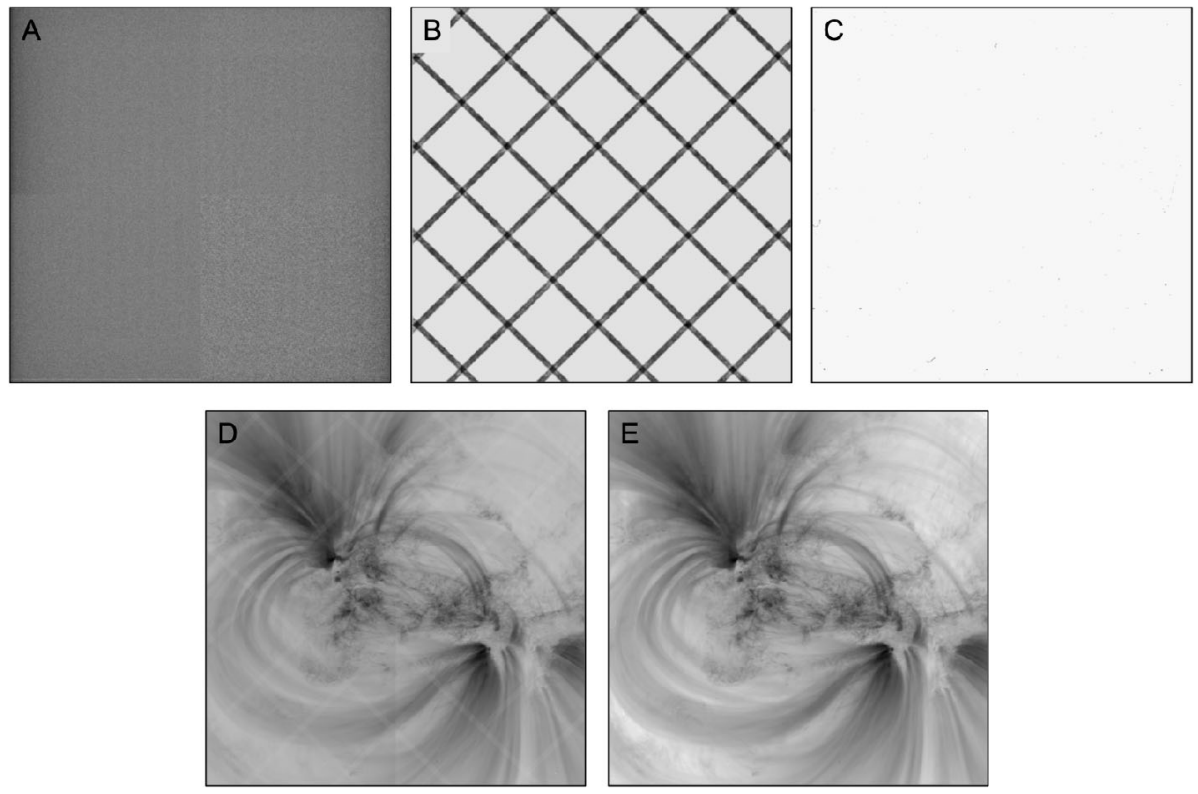

Figure 12 The upper panels show the A) master dark, B) master flat, and C) and bad pixel map, respectively. The bottom panel shows an example of $\mathbf{D})$ the raw Hi-C data (rotated and cropped) and $\mathbf{E}$ ) a calibrated image. Figures $\mathrm{D}$ and $\mathrm{E}$ are plotted with an inverse color table.

Table 4 Hi-C 2.1 flight data summary.

\begin{tabular}{|c|c|c|c|}
\hline Channel & $172 \AA$ & Image Size & $2064 \times 2048$ \\
\hline Launch Date & May 29, 2018 & Field of View & $4.44^{\prime} \times 4.40^{\prime}$ \\
\hline Data Acquisition Time & $18: 56: 22-19: 01: 57$ & Pointing & $\left(-114^{\prime \prime}, 259^{\prime \prime}\right) \pm 0.3^{\prime \prime}$ \\
\hline Camera Gain & $2.5 \pm 0.02 \mathrm{elec} \mathrm{DN}^{-1}$ & Roll & $0.985 \pm 0.005^{\circ} \mathrm{CW}$ \\
\hline Camera Noise ${ }^{*}$ : & & Exposure Time & $2 \mathrm{~s}$ \\
\hline NE Quad & $4.0 \mathrm{DN}$ & Full Data Set: & \\
\hline NW Quad & $3.4 \mathrm{DN}$ & No. of Images & 78 \\
\hline SE Quad & $5.5 \mathrm{DN}$ & Cadence & $4.4 \mathrm{~s}$ \\
\hline SW Quad & $3.6 \mathrm{DN}$ & Low Jitter Set: & \\
\hline Plate Scale & $0.129^{\prime \prime} \mathrm{pixel}^{-1}$ & No. of Images & 36 \\
\hline Resolution & $<0.47^{\prime \prime}$ (low jitter images only) & Cadence & $4.4 \mathrm{~s}$ (periodic $\sim 20 \mathrm{~s}$ gaps) \\
\hline
\end{tabular}

*Solar coordinates; solar north at top of frame.

of bias-subtracted darks taken during the ascent phase of the flight. The results per quadrant of this set range from $\sim 3.6$ to $5.5 \mathrm{DN}$ (i.e., $\sim 9-13.8 \mathrm{e}^{-}$.)

The noise of the master dark (created from a median of these images as described above) is $\sim 1 \mathrm{DN}$ per quadrant, therefore not adding any significant noise to the science images during processing (described below).

A summary of the flight data parameters, as described in the preceding sections, is provided in Table 4. 


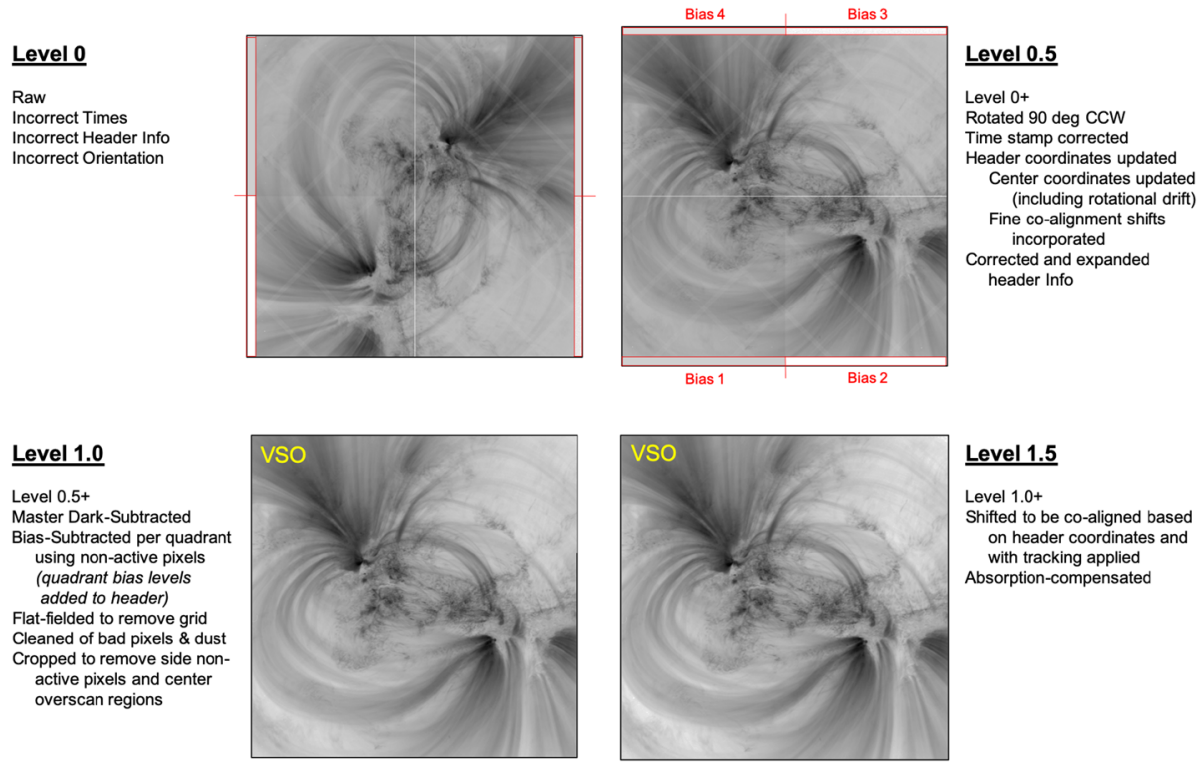

Figure 13 Summary of the processed data levels and availability through the VSO.

Data sets have been generated at progressive levels of processing (see Figure 13). Level 0.5 data was rotated to place solar north along the top of each frame, and the FITS file header information was updated (e.g., corrected time and pointing). Level 1.0 data is biasand dark-subtracted, flat-fielded, bad pixel-corrected, and cropped to remove non-active and overscan regions. Level 1.5 data is shifted to be co-aligned (with target tracking applied), and the intensity levels are compensated for atmospheric absorption.

Levels 1.0 and 1.5 have been distributed by the science team via the Virtual Solar Observatory (VSO). Additional information on Hi-C 2.1 image processing is available in the User Guide distributed with the data. For these two levels, an accompanying low jitter set is provided which excludes the frames most affected by motion blur from the rocket.

\subsection{Co-Observations}

The Hi-C 2.1 flight was coordinated with several other ground- and space-based solar observing observatories, including IRIS, three telescopes onboard the Hinode satellite, SDO/AIA, the National Solar Observatory Interferometric BIdimensional Spectropolarimeter (NSO/IBIS), the Nuclear Spectroscopic Telescope Array (NuSTAR), the Big Bear Solar Observatory, the Owens Valley Radio Observatory, and the Swedish Solar Telescope. Due to various circumstances, particularly regarding weather, the most successful coordinations resulted from IRIS, the Hinode suite, AIA, IBIS, and NuSTAR.

The primary science driver to connect chromospheric and coronal heating events necessitated key overlapping data from IRIS. Fortunately, coordinations with IRIS were highly successful (IRIS OBSID 3600104031), providing high-resolution images and spectra of the AR core and loops within the Hi-C 2.1 FOV (Figure 14). 

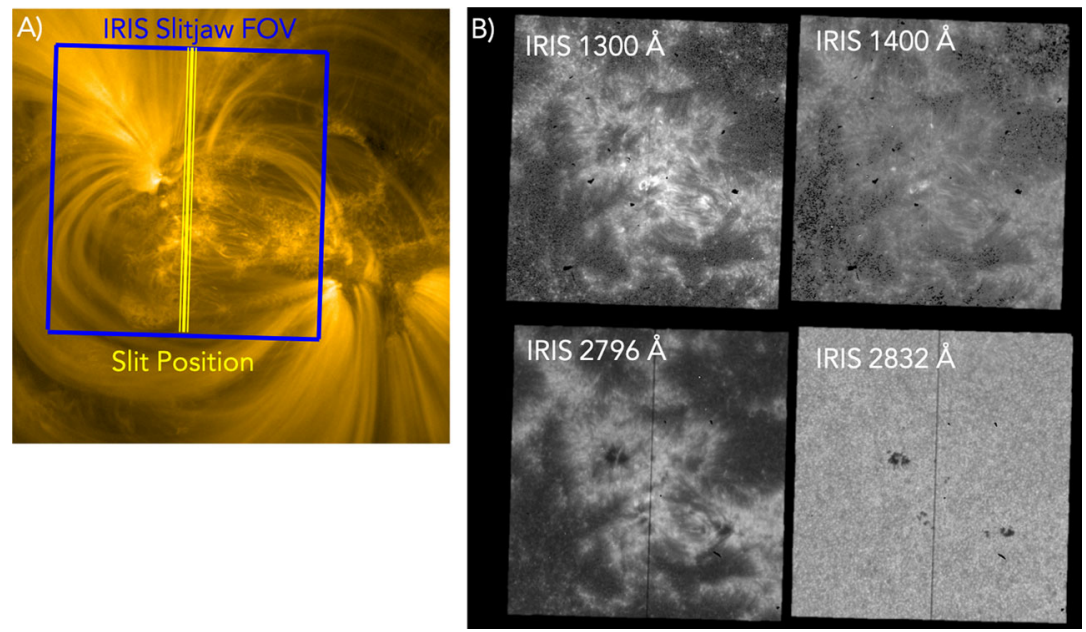

Figure 14 Co-temporal IRIS observations. A) IRIS slitjaw FOV and slit position overlaid onto the Hi-C 2.1 FOV. B) Example IRIS slitjaw images from the coordinated set.

$\mathrm{Hi}-\mathrm{C} 172$

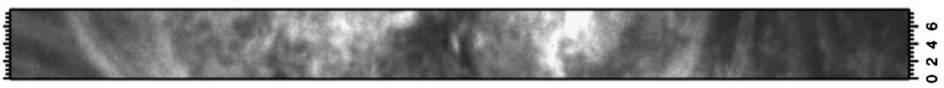

Si IV

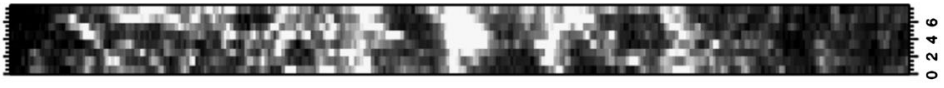

C II

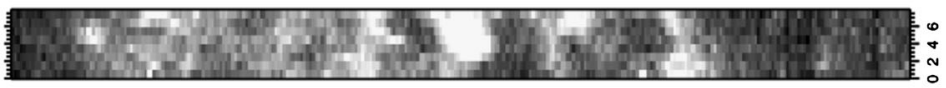

Mg II k

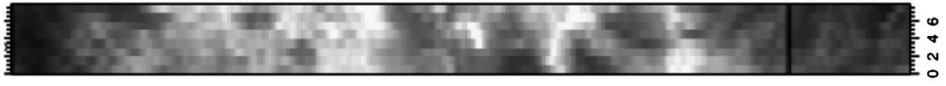

Photosphere

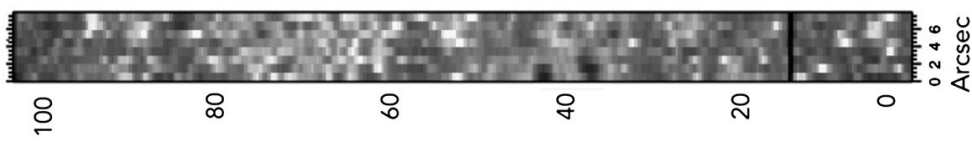

Arcsec

Figure 15 Sparse IRIS rasters, summed spatially and spectrally by 2, of the photosphere, $\mathrm{Mg}$ II $\mathrm{k}$ core, C II, and Si IV lines along with the Hi-C 2.1 intensity extracted to match the 8 arcsec width of the IRIS raster. Full spectral information of the IRIS line profiles is available at each spatial pixel.

The IRIS spectroheliograms (Figure 15) were optimized to obtain fast rasters of a region of $8^{\prime \prime} \times 175^{\prime \prime}$ within $25 \mathrm{~s}$ (using exposure times of $2 \mathrm{~s}$ and sparse $1^{\prime \prime}$ steps). To maximize faint transition-region signals, the data was summed spatially and spectrally by 2 , so that the resulting resolution is $0.66^{\prime \prime}$ along the slit (sampled at $0.33^{\prime \prime}$ ) with $1^{\prime \prime}$ steps across the slit. At each slit location, spectral information is available for the C II $1335 \AA$, O I $1356 \AA$, Si IV $1394 \AA$, Si IV $1042 \AA$, and Mg II $2796 \AA$ k and Mg II $2803 \AA \mathrm{h}$ lines. This unique combined data set provides the first sub-arcsec resolution data set covering the full solar atmosphere from the photosphere, through the chromosphere and transition region into the corona. 


\section{Conclusions}

The third flight of the Hi-C sounding rocket, Hi-C 2.1, has resulted in the highest resolution data of the cool corona and transition region ever taken at $172 \AA$. The full set of calibrated data from the flight are now available on the VSO. Together with the coordinated observations, this data set provides a unique view of this important region, which will enhance our scientific knowledge of the coupling of mass and energy between the chromosphere and the corona. Despite the fact that the data only span $\sim 5 \mathrm{~min}$ in time, the scientific advancements made by the first flight, and the data analysis already begun on this second set of data prove that the fine-scale structure of this region is key to our understanding of the solar atmosphere.

Acknowledgements We acknowledge the High-resolution Coronal Imager (Hi-C 2.1) instrument team for making the second re-flight data available under NASA Heliophysics Technology and Instrument Development for Science (HTIDS) Low Cost Access to Space (LCAS) program (proposals HTIDS14_2-0048 and HTIDS17_2-0033). MSFC/NASA led the mission with partners including the Smithsonian Astrophysical Observatory, the University of Central Lancashire, and Lockheed Martin Solar and Astrophysics Laboratory. Hi-C 2.1 was launched out of the White Sands Missile Range on 2018 May 29. The AIA data are courtesy of NASA/SDO and the AIA Science Team. S.K.T. gratefully acknowledges support by NASA contracts NNG09FA40C (IRIS), and NNM07AA01C (Hinode). The work of DHB was performed under contract to the Naval Research Laboratory and was funded by the NASA Hinode program.

Disclosure of Potential Conflicts of Interest The authors declare that they have no conflicts of interest.

Publisher's Note Springer Nature remains neutral with regard to jurisdictional claims in published maps and institutional affiliations.

Open Access This article is distributed under the terms of the Creative Commons Attribution 4.0 International License (http://creativecommons.org/licenses/by/4.0/), which permits unrestricted use, distribution, and reproduction in any medium, provided you give appropriate credit to the original author(s) and the source, provide a link to the Creative Commons license, and indicate if changes were made.

\section{References}

Alexander, C.E., Walsh, R.W., Régnier, S., Cirtain, J., Winebarger, A.R., Golub, L., Kobayashi, K., Platt, S., Mitchell, N., Korreck, K., DePontieu, B., DeForest, C., Weber, M., Title, A., Kuzin, S.: 2013, Antiparallel EUV flows observed along active region filament threads with Hi-C. Astrophys. J. Lett. 775, L32. DOI. ADS.

Alpert, S.E., Tiwari, S.K., Moore, R.L., Winebarger, A.R., Savage, S.L.: 2016, Hi-C observations of sunspot penumbral bright dots. Astrophys. J. 822, 35. DOI. ADS.

Berger, T.E., De Pontieu, B., Fletcher, L., Schrijver, C.J., Tarbell, T.D., Title, A.M.: 1999, What is moss? Solar Phys. 190, 409. DOI. ADS.

Brooks, D.H., Warren, H.P., Ugarte-Urra, I., Winebarger, A.R.: 2013, High spatial resolution observations of loops in the solar corona. Astrophys. J. Lett. 772, L19. DOI. ADS.

Cheung, M.C.M., Boerner, P., Schrijver, C.J., Testa, P., Chen, F., Peter, H., Malanushenko, A.: 2015, Thermal diagnostics with the atmospheric imaging assembly on board the solar dynamics observatory: a validated method for differential emission measure inversions. Astrophys. J. 807, 143. DOI. ADS.

Cirtain, J.W., Golub, L., Winebarger, A.R., De Pontieu, B., Kobayashi, K., Moore, R.L., Walsh, R.W., Korreck, K.E., Weber, M., McCauley, P., Title, A., Kuzin, S., Deforest, C.E.: 2013, Energy release in the solar corona from spatially resolved magnetic braids. Nature 493, 501. DOI. ADS.

De Pontieu, B., Tarbell, T., Erdélyi, R.: 2003, Correlations on arcsecond scales between chromospheric and transition region emission in active regions. Astrophys. J. 590, 502. DOI. ADS.

De Pontieu, B., Berger, T.E., Schrijver, C.J., Title, A.M.: 1999, Dynamics of transition region 'Moss' at high time resolution. Solar Phys. 190, 419. DOI. ADS. 
De Pontieu, B., McIntosh, S., Hansteen, V.H., Carlsson, M., Schrijver, C.J., Tarbell, T.D., Title, A.M., Shine, R.A., Suematsu, Y., Tsuneta, S., Katsukawa, Y., Ichimoto, K., Shimizu, T., Nagata, S.: 2007, A tale of two spicules: the impact of spicules on the magnetic chromosphere. Publ. Astron. Soc. Japan 59, S655. DOI. ADS.

De Pontieu, B., McIntosh, S.W., Carlsson, M., Hansteen, V.H., Tarbell, T.D., Boerner, P., Martinez-Sykora, J., Schrijver, C.J., Title, A.M.: 2011, The origins of hot plasma in the solar corona. Science 331, 55. DOI. ADS.

De Pontieu, B., Title, A.M., Lemen, J.R., Kushner, G.D., Akin, D.J., Allard, B., Berger, T., Boerner, P., Cheung, M., Chou, C., Drake, J.F., Duncan, D.W., Freeland, S., Heyman, G.F., Hoffman, C., Hurlburt, N.E., Lindgren, R.W., Mathur, D., Rehse, R., Sabolish, D., Seguin, R., Schrijver, C.J., Tarbell, T.D., Wülser, J.-P., Wolfson, C.J., Yanari, C., Mudge, J., Nguyen-Phuc, N., Timmons, R., van Bezooijen, R., Weingrod, I., Brookner, R., Butcher, G., Dougherty, B., Eder, J., Knagenhjelm, V., Larsen, S., Mansir, D., Phan, L., Boyle, P., Cheimets, P.N., DeLuca, E.E., Golub, L., Gates, R., Hertz, E., McKillop, S., Park, S., Perry, T., Podgorski, W.A., Reeves, K., Saar, S., Testa, P., Tian, H., Weber, M., Dunn, C., Eccles, S., Jaeggli, S.A., Kankelborg, C.C., Mashburn, K., Pust, N., Springer, L., Carvalho, R., Kleint, L., Marmie, J., Mazmanian, E., Pereira, T.M.D., Sawyer, S., Strong, J., Worden, S.P., Carlsson, M., Hansteen, V.H., Leenaarts, J., Wiesmann, M., Aloise, J., Chu, K.-C., Bush, R.I., Scherrer, P.H., Brekke, P., Martinez-Sykora, J., Lites, B.W., McIntosh, S.W., Uitenbroek, H., Okamoto, T.J., Gummin, M.A., Auker, G., Jerram, P., Pool, P., Waltham, N.: 2014, The Interface Region Imaging Spectrograph (IRIS). Solar Phys. 289, 2733. DOI. ADS.

De Pontieu, B., De Moortel, I., Martinez-Sykora, J., McIntosh, S.W.: 2017, Observations and numerical models of solar coronal heating associated with spicules. Astrophys. J. 845(2), L18. DOI. ADS.

Fletcher, L., De Pontieu, B.: 1999, Plasma diagnostics of transition region "moss" using SOHO/CDS and TRACE. Astrophys. J. 520, L135. DOI. ADS.

Kobayashi, K., Cirtain, J., Winebarger, A.R., Korreck, K., Golub, L., Walsh, R.W., De Pontieu, B., DeForest, C., Title, A., Kuzin, S., Savage, S., Beabout, D., Beabout, B., Podgorski, W., Caldwell, D., McCracken, K., Ordway, M., Bergner, H., Gates, R., McKillop, S., Cheimets, P., Platt, S., Mitchell, N., Windt, D.: 2014, The high-resolution coronal imager (Hi-C). Solar Phys. 289, 4393. DOI. ADS.

Lemen, J.R., Title, A.M., Akin, D.J., Boerner, P.F., Chou, C., Drake, J.F., Duncan, D.W., Edwards, C.G., Friedlaender, F.M., Heyman, G.F., Hurlburt, N.E., Katz, N.L., Kushner, G.D., Levay, M., Lindgren, R.W., Mathur, D.P., McFeaters, E.L., Mitchell, S., Rehse, R.A., Schrijver, C.J., Springer, L.A., Stern, R.A., Tarbell, T.D., Wuelser, J.-P., Wolfson, C.J., Yanari, C., Bookbinder, J.A., Cheimets, P.N., Caldwell, D., Deluca, E.E., Gates, R., Golub, L., Park, S., Podgorski, W.A., Bush, R.I., Scherrer, P.H., Gummin, M.A., Smith, P., Auker, G., Jerram, P., Pool, P., Soufli, R., Windt, D.L., Beardsley, S., Clapp, M., Lang, J., Waltham, N.: 2012, The Atmospheric Imaging Assembly (AIA) on the Solar Dynamics Observatory (SDO). Solar Phys. 275, 17. DOI. ADS.

Martens, P.C.H., Kankelborg, C.C., Berger, T.E.: 2000, On the nature of the "moss" observed by TRACE. Astrophys. J. 537, 471. DOI. ADS.

Peres, G., Reale, F., Golub, L.: 1994, Loop models of low coronal structures observed by the Normal Incidence X-Ray Telescope (NIXT). Astrophys. J. 422, 412. DOI. ADS.

Peter, H., Bingert, S., Klimchuk, J.A., de Forest, C., Cirtain, J.W., Golub, L., Winebarger, A.R., Kobayashi, K., Korreck, K.E.: 2013, Structure of solar coronal loops: from miniature to large-scale. Astron. Astrophys. 556, A104. DOI. ADS.

Pontin, D.I., Janvier, M., Tiwari, S.K., Galsgaard, K., Winebarger, A.R., Cirtain, J.W.: 2017, Observable signatures of energy release in braided coronal loops. Astrophys. J. 837, 108. DOI. ADS.

Régnier, S., Alexander, C.E., Walsh, R.W., Winebarger, A.R., Cirtain, J., Golub, L., Korreck, K.E., Mitchell, N., Platt, S., Weber, M., De Pontieu, B., Title, A., Kobayashi, K., Kuzin, S., DeForest, C.E.: 2014, Sparkling extreme-ultraviolet bright dots observed with Hi-C. Astrophys. J. 784, 134. DOI. ADS.

Schmelz, J.T., Reames, D.V., von Steiger, R., Basu, S.: 2012, Composition of the solar corona, solar wind, and solar energetic particles. Astrophys. J. 755, 33. DOI. ADS.

Testa, P., De Pontieu, B., Martínez-Sykora, J., DeLuca, E., Hansteen, V., Cirtain, J., Winebarger, A., Golub, L., Kobayashi, K., Korreck, K., Kuzin, S., Walsh, R., DeForest, C., Title, A., Weber, M.: 2013, Observing coronal nanoflares in active region moss. Astrophys. J. 770, L1. DOI. ADS.

Thalmann, J.K., Tiwari, S.K., Wiegelmann, T.: 2014, Force-free field modeling of twist and braiding-induced magnetic energy in an active-region corona. Astrophys. J. 780, 102. DOI. ADS.

Tiwari, S.K., Alexander, C.E., Winebarger, A.R., Moore, R.L.: 2014, Trigger mechanism of solar subflares in a braided coronal magnetic structure. Astrophys. J. Lett. 795, L24. DOI. ADS.

Tiwari, S.K., Moore, R.L., Winebarger, A.R., Alpert, S.E.: 2016, Transition-region/coronal signatures and magnetic setting of sunspot penumbral jets: Hinode (SOT/FG), Hi-C, and SDO/AIA observations. Astrophys. J. 816, 92. DOI. ADS. 
Voronov, D.L., Anderson, E.H., Cambie, R., Gullikson, E.M., Salmassi, F., Warwick, T., Yashchuk, V.V., Padmore, H.A.: 2011, Roughening and smoothing behavior of A1/Zr multilayers grown on flat and saw-tooth substrates. In: Society of Photo-Optical Instrumentation Engineers (SPIE) Conference Series 8139, 81390B. DOI. ADS.

Warren, H.P., Winebarger, A.R., Mariska, J.T., Doschek, G.A., Hara, H.: 2008, Observation and modeling of coronal "Moss" with the EUV imaging spectrometer on Hinode. Astrophys. J. 677, 1395. DOI. ADS.

Windt, D.L.: 2015, EUV multilayer coatings for solar imaging and spectroscopy. In: Solar Physics and Space Weather Instrumentation VI, Society of Photo-Optical Instrumentation Engineers (SPIE) Conference Series 9604, 96040P. DOI. ADS.

Windt, D.L., Waskiewicz, W.K.: 1994, Multilayer facilities required for extreme-ultraviolet lithography. J. Vac. Sci. Technol., B Microelectron. Nanometer Struct. 12, 3826. DOI. ADS.

Winebarger, A.R., Walsh, R.W., Moore, R., De Pontieu, B., Hansteen, V., Cirtain, J., Golub, L., Kobayashi, K., Korreck, K., DeForest, C., Weber, M., Title, A., Kuzin, S.: 2013, Detecting nanoflare heating events in subarcsecond inter-moss loops using Hi-C. Astrophys. J. 771, 21. DOI. ADS.

Winebarger, A.R., Cirtain, J., Golub, L., DeLuca, E., Savage, S., Alexander, C., Schuler, T.: 2014, Discovery of finely structured dynamic solar corona observed in the Hi-C telescope. Astrophys. J. Lett. 787, L10. DOI. ADS.

Young, I.D.U.o.T., Gerbrands, J.D.U.o.T., van Vliet, L.D.U.: 2011, Fundamentals of image processing. In: Optical and Digital Image Processing: Fundamentals and Applications 71. ISBN: 9783527635245. DOI.

Zhong, Q., Li, W., Zhang, Z., Zhu, J., Huang, Q., Li, H., Wang, Z., Jonnard, P., Le Guen, K., André, J.-M., Zhou, H., Huo, T.: 2012, Optical and structural performance of the $\mathrm{Al}(1 \% \mathrm{wtSi}) / \mathrm{Zr}$ reflection multilayers in the $17-19 \mathrm{~nm}$ region. Opt. Express 20, 10692. DOI. ADS. 